\title{
Myelopathy due to bilateral symmetrical dumbbell cervical ganglioneuroma in a pediatric neurofibromatosis type 1 patient and rigid posterior instrumentation-a case report
}

Omer Batu Hergunsel ${ }^{1}$, Fatih Demir ${ }^{1}$, Mehmet Mustafa Akin $^{2}$ and Metin Kaplan ${ }^{1 *}$

\begin{abstract}
Background: Although neurofibromatosis type 1 (NF1) is a common genetical disorder, symptoms due to neural involvement are rare among infants and children. Ganglioneuromas are slow-growing tumors and can be seen in patients with NF1. However, bilateral symmetrical involvement is very rarely reported in the literature. We present a rare pediatric case with NF1 who presented with upper cervical bilateral symmetrical ganglioneuroma.

Case presentation: A 7-year-old male presented with progressive gait disturbance, falls, and bilateral limb and trunk numbness that started several weeks ago. Physical examination revealed generalized café au lait spots, neurofibromas, and axillary and inguinal freckling. Family history for NF1 was positive. Neurological examination showed slight paraparesis and hypoesthesia of the upper extremities with C4-C5 dermatomas being more distinct. Cervical magnetic resonance imaging (MRI) revealed contrast enhancing dumbbell-shaped symmetrical lesions originating from both C3 nerve roots extending to C2-C5 vertebral body levels. There was a significant spinal cord compression causing myelopathy as well as dystrophic changes and kyphotic deformity at those levels. The patient was operated and the lesions were totally excised via C2-3-4 laminoplasty. Histopathological evaluation reported the lesion as ganglioneuroma. Following the surgery for excision of the lesions, the patient presented with rapidly progressing kyphotic deformity and underwent rigid craniocervical fusion.

Conclusion: Ganglioneuromas are seen among children, tend to occur in the mediastinum, abdomen, and retroperitoneal area, and may accompany NF1. Cervical spinal ganglioneuroma, however, is a very rare entity. Coexistence of NF1 and bilateral symmetrical cervical ganglioneuroma is highly unusual. Like neurofibromas, ganglioneuromas are slow-growing tumors and therefore they are not expected to cause myelopathy and related symptoms during childhood. Symmetrical nature of the lesions results in myelopathy and early onset of symptoms. Due to the risk of postoperative increase of kyphotic angulation, laminoplasty should be considered. Although laminoplasty alone does not prevent the progression of kyphotic changes, the procedure may allow the use of sublaminar wires and laminar hooks for future surgeries to correct the deformity.
\end{abstract}

Keywords: Neurofibromatosis type 1, Ganglioneuroma, Laminoplasty, Craniocervical fusion

\footnotetext{
*Correspondence: mtkaplan02@yahoo.com.tr

'Department of Neurosurgery, Firat University School of Medicine, Elazig,

Turkey

Full list of author information is available at the end of the article
} 


\section{Background}

Neurofibromatosis type 1 is one of the most common genetically disorders and has a wide clinical spectrum. Although the disease presents with multisystem involvement, neural involvement determines the prognosis. Besides the involvement of soft tissue, bone dysplasias, pseudarthrosis, scoliosis, and kyphotic deformities are remarkable in neurofibromatosis type 1 (NF1) [4, 5, 14]. Symptoms due to neural involvement are very rare among infants and children [6, 12]. Ganglioneuromas are slow-growing benign tumors and can be seen in patients with NF1. However, spinal localization, especially bilateral symmetrical involvement, is very rarely reported in the literature [7, 11, 13]. Cervical spinal deformities are usually asymptomatic among NF1 patients, and there is rarely need for surgical correction [2, 14]. On the other hand, when needed, surgical management of cervical deformities is challenging in children [3].

We present the rare case of a 7-year-old male with NF1 who presented with an upper cervical bilateral symmetrical ganglioneuroma. The patient discussed had early symptoms due to myelopathy caused by bilateral mass lesions. After the surgery for the removal of the lesions, he had to undergo additional rigid craniocervical fusion for rapidly progressing kyphotic deformity.

\section{Case presentation}

A 7-year-old male presented with progressive gait disturbance, falls, and bilateral limb and trunk numbness that started several weeks ago. Neck movements were restricted due to the pain. Physical examination revealed generalized café au lait spots, generalized neurofibromas with a plexiform neurofibroma on the left side of the neck, and axillary and inguinal freckling. Family history for NF1 was positive, and the patient was already under follow-up by pediatrics. Neurological examination showed slight paraparesis and hypoesthesia of the upper extremities with C4-C5 dermatomas being more distinct. Deep tendon reflexes were hyperactive bilaterally with a positive Babinski sign on both sides. Cervical magnetic resonance imaging revealed contrast enhancing dumbbell-shaped symmetrical lesions originating from both $\mathrm{C} 3$ nerve roots extending to $\mathrm{C} 2-\mathrm{C} 5$ vertebral body levels. There was a significant spinal cord compression causing myelopathy as well as dystrophic changes and kyphotic deformity at those levels. Spinal canal and the neural foramina showed a significant widening (Fig. 1a, b).

The patient was operated in the prone position, and the lesions were totally excised via C2-3-4 laminoplasty (Fig. 2a). Postoperatively, the patient had no neurological deficit except hypoesthesia of C4-5 dermatomas bilaterally. Gait disorder and generalized numbness recovered fully, and he was discharged with a rigid cervical collar. Histopathological evaluation reported the lesion as ganglioneuroma (Fig. 3).

On 2-week follow-up postoperatively, the patient had worsening of head and neck posture and complaints of generalized numbness. The family reported that he did not regularly use the collar due to discomfort. Cervical MRI showed significant progression of kyphotic deformity (Fig. 2b). Following gradual traction to correct kyphotic deformity under general anesthesia, a halo fixation device was applied (Fig. 4a). After application of the halo vest, the patient was free of numbness and neck pain complaints. Four months after, the device was removed. During the follow-up, radiological assessment showed recurrence of deformity with no clinical

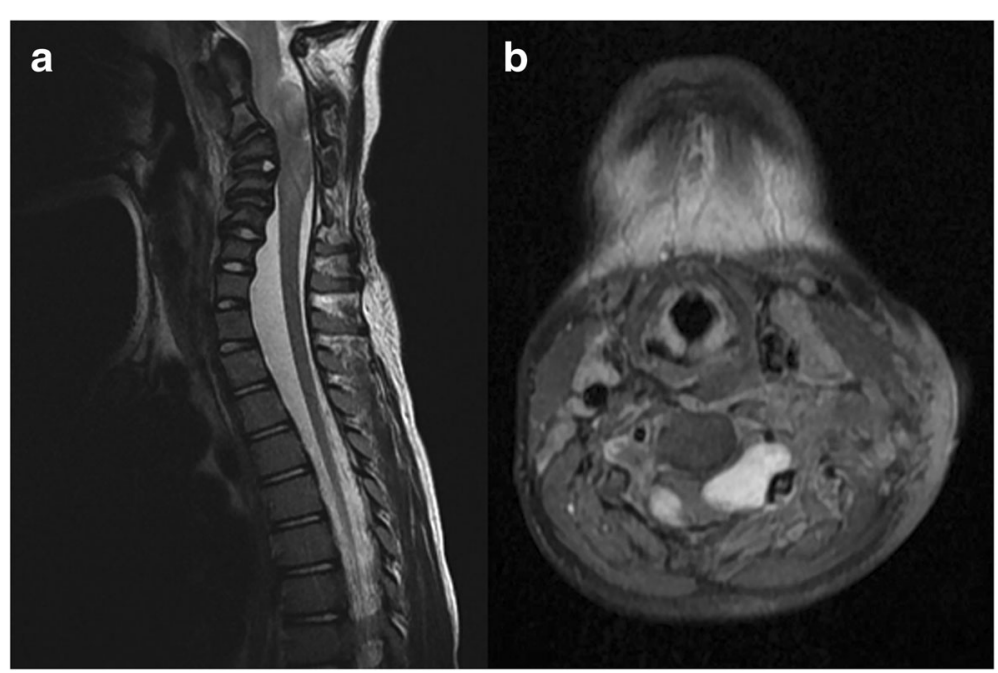

Fig. 1 a T2-weighted sagittal MRI showing significant compression of the spinal cord, dystrophic changes, and kyphotic deformity of cervical vertebrae and widening of spinal canal. b Bilateral dumbbell-shaped lesions on contrast-enhanced T1 weighted axial MRI 


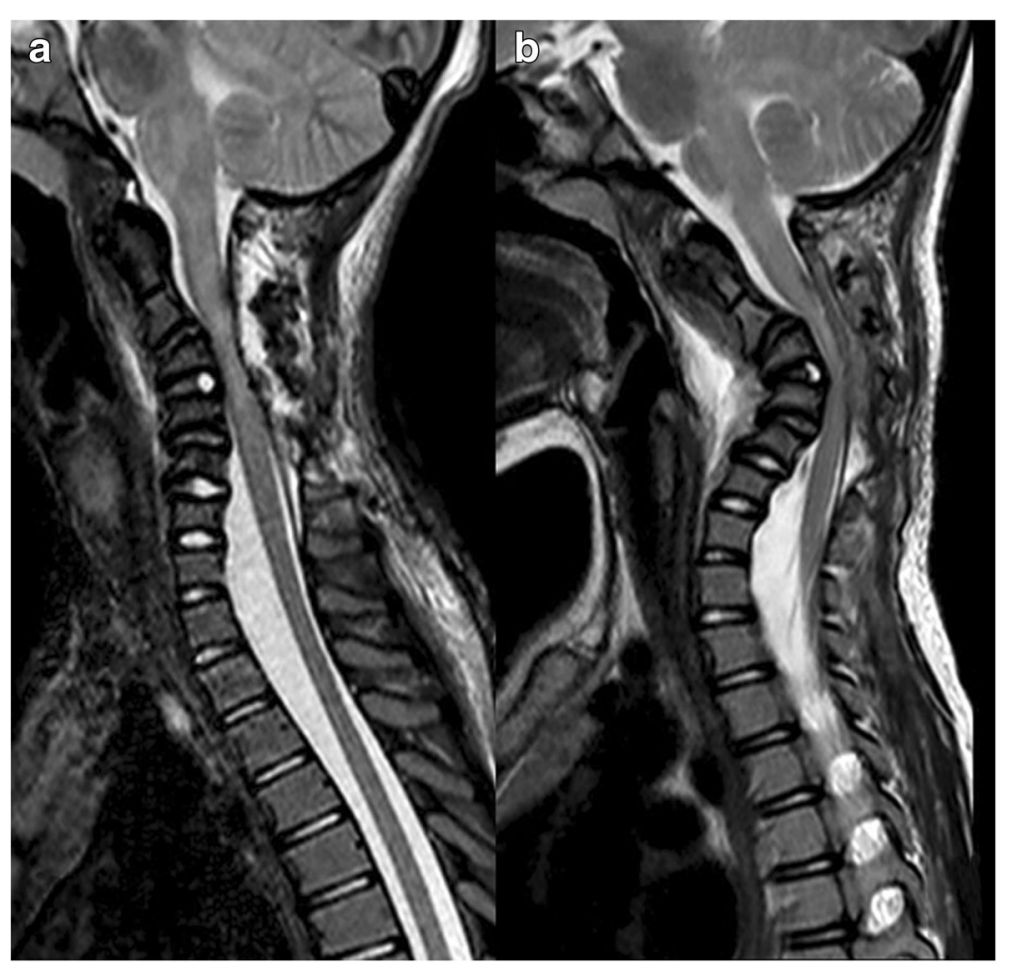

Fig. 2 a Postoperative T2-weighted sagittal MRI showing total resection of the lesions. b T2-weighted sagittal MRI demonstrating progression of kyphotic deformity

symptoms (Fig. 4b). The patient underwent additional rigid craniocervical fusion using laminar hooks and sublaminar wires.

\section{Stabilization surgery}

Under general anesthesia and in a supine position, kyphotic deformity was gradually corrected as possible. A significant correction of the deformity was only possible with an overextension of the head (Fig. 4a), which was not a suitable posture to minimally affect activities of daily living. Therefore, the extent of reduction was revised in order to maintain an appropriate posture (i.e., gaze parallel to the ground) for rigid stabilization. Then the patient was put in a prone position with a Mayfield skull clamp, and the reduction angle was reconfirmed via fluoroscopy.

Surgical exposure revealed total bony fusion at the levels of previous laminoplasty. C4-T1 laminar hooks and $\mathrm{C} 3$ sublaminar wires were used to maintain occipitocervical stabilization. Due to their position, $\mathrm{C} 1$ and $\mathrm{C} 2$ vertebral bodies were not connected to rods via hooks or wire (Fig. 5a).

There was no complication, and the patient was neurologically intact save for hypoethesia of C4-5 dermatomas bilaterally. On 1-year follow-up, his neurological status remained stable and cervical X-rays demonstrated no change in cervical spinal alignment postoperatively (Fig. 5b).

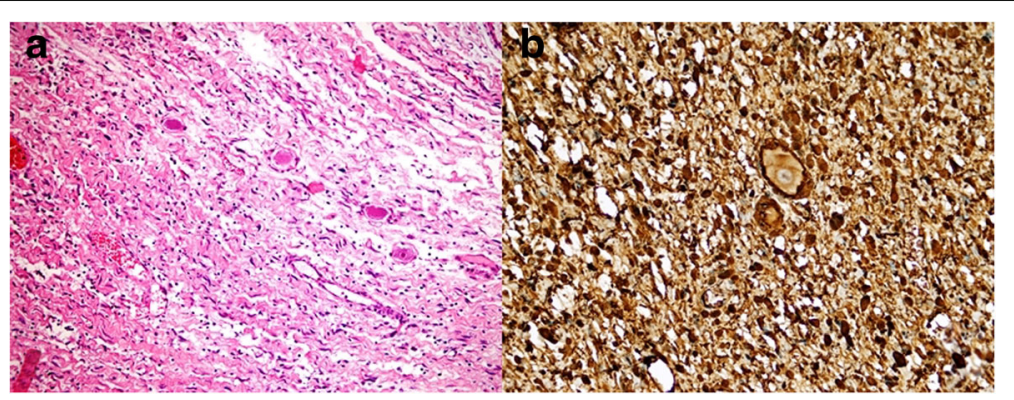

Fig. 3 a Admixture of ganglion cells and Schwann cells (hematoxylin and eosin staining, $\times 10$ ). $\mathbf{b}$ Admixture of ganglion cells and Schwann cells (S100 staining, $\times 20$ ) 


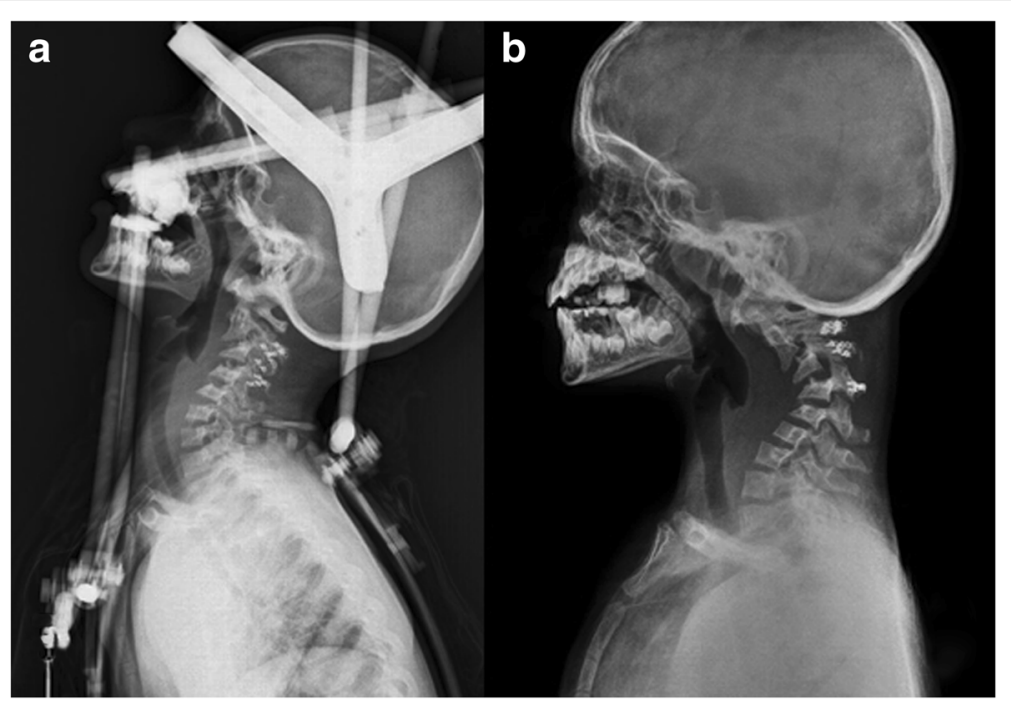

Fig. 4 a Lateral cervical spine X-ray showing correction of the deformity with Halo fixation device. $\mathbf{b}$ Lateral cervical spine X-ray showing reincrease of cervical kyphosis after removal of halo vest

\section{Conclusion}

NF1 is an autosomal dominant disease with an incidence rate of 1 per 3000 persons which is characterized with generalized neurofibromas. Although spinal involvement is not uncommon for neurofibromas, myelopathy and related symptoms are very rare during childhood [12]. Our case was a 7-year-old and presented with neurological symptoms due to myelopathy caused by mass lesions. Moreover, dumbbell-shaped lesions of the upper cervical region are extremely rare for that age and seldom cause myelopathy and relevant symptoms $[6,12]$. It was remarkable for our case that bilateral symmetrical dumbbell-shaped lesions were identified on MRI with absence of any lesion at other levels of the spinal canal. Despite this atypical presentation, the history of NF1 suggested the lesion to be a neurofibroma at first glance. Histopathological examination resulted in a diagnosis of ganglioneuroma. Ganglioneuromas are more commonly

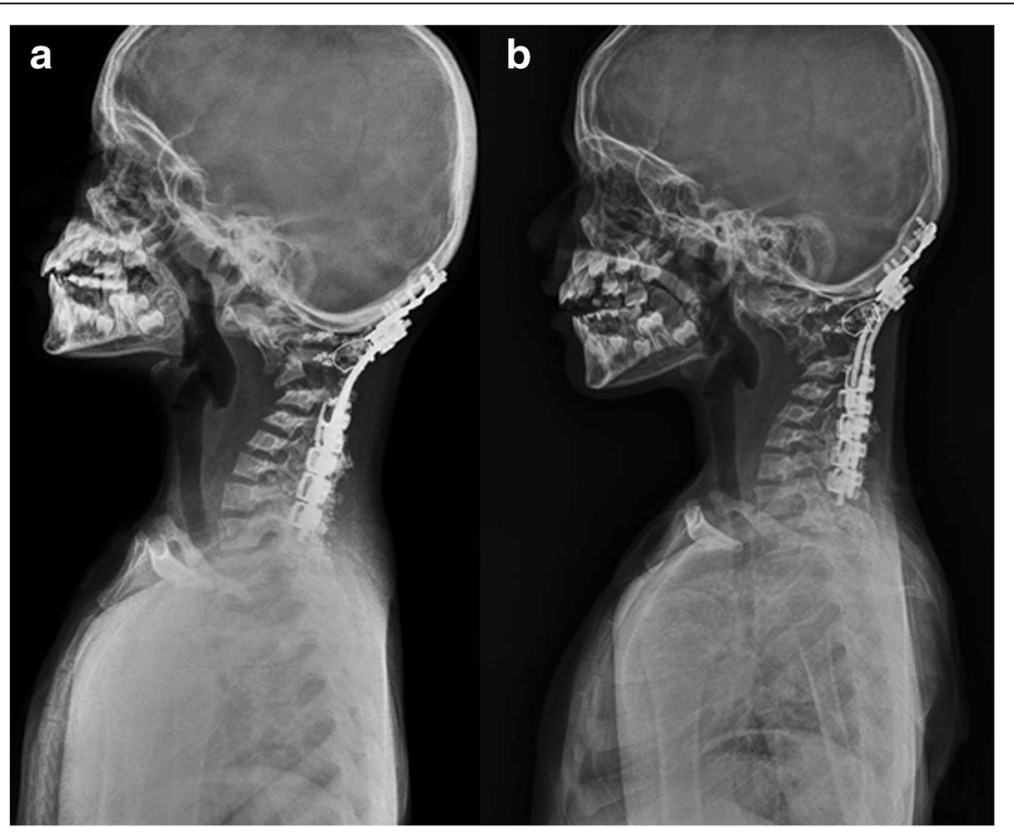

Fig. 5 a Long segment rigid fixation between occiput and $\mathrm{T} 1$ vertebral body with sublaminar wires and laminar hooks. b Lateral cervical X-ray at 1 -year follow-up demonstrating that there is no progression of angulation 
seen among children and young adults, tend to occur in the mediastinum, abdomen, and retroperitoneal area, and may accompany NF1. Cervical spinal ganglioneuroma, however, is a very rare entity. Co-existence of NF1 and bilateral symmetrical cervical ganglioneuroma is highly unusual $[7,11,13]$. Differential diagnosis should include bilateral symmetrical dumbbell neurofibroma, another very rare condition in patients with NF1.

Like neurofibromas, ganglioneuromas are slow-growing tumors and therefore they are not expected to cause myelopathy and related symptoms during childhood [7, 12, 13]. Despite having a widened spinal canal, myelopathy and related symptoms were the initial presentation of our case. We suggest that the bilateral and symmetrical nature of the lesion had an effect on that.

NF1 patients usually have pathological changes in the bone and ligament structures such as widened foramina, dysplasia, scalloping of the vertebral body, kyphotic deformities, and subluxations. Significant dystrophic changes in the upper cervical region and kyphotic deformity were remarkable in our case as well as widening of spinal canal and neural foramina. For such cases, cervical spinal involvement may be asymptomatic and therefore close follow-up is advised [2, 8]. Our case, however, underwent decompression surgery due to symptoms of myelopathy.

Cervical kyphotic deformity following laminectomy is more common among children [1]. It is obvious that NF1 patients who already have dystrophic and kyphotic changes are more vulnerable to postlaminectomy $\mathrm{ky}$ phosis. Therefore, a laminoplasty procedure was applied although controversial on whether it prevented kyphotic deformity $[9,10]$. However, due to the patient's age, initial surgery cannot be combined with internal stabilization. Follow-up with a rigid brace was planned. During postoperative follow-up, kyphotic deformity progressed significantly. Kyphotic deformity was reduced with careful traction under general anesthesia, and a halo fixation vest was applied. Radiological follow-up after the removal of the device showed a reincrease of the kyphotic angle, and surgical rigid fixation was decided.

For the pediatric patients, surgical armaments for cervical stabilization are limited, and especially for the age group of our case, stabilization of cervical kyphosis and long-segment fixation is very challenging. In pediatric patients, sublaminar wires and laminar hooks are usually preferred for stabilization procedures [3]. We used sublaminar wires and laminar hooks to establish long-segment occipitocervical stabilization including T1 vertebra. We noted that the widening of the spinal canal provided an advantage for safely placing laminar hooks. In addition to this, bony fusion was established across laminoplasty segments as expected, which enabled C3 sublaminar wiring and $\mathrm{C} 4$ hook placement. As a result, laminoplasty procedure and widening of the spinal canal allowed for a relatively safe and effective stabilization procedure.

In conclusion, presence of dumbbell-shaped symmetrical lesions in pediatric NF1 patients should lead to a differential diagnosis including ganglioneuroma. The symmetrical nature of the lesions results in myelopathy and early onset of symptoms. Due to the risk of postoperative increase of kyphotic angulation, laminoplasty should be considered. Although laminoplasty alone does not prevent the progression of kyphotic changes, the procedure may allow the use of sublaminar wires and laminar hooks for future surgeries to correct the deformity.

\section{Abbreviations \\ MRI: Magnetic resonance imaging; NF1: Neurofibromatosis type 1 \\ Acknowledgements \\ Not applicable \\ Funding \\ No financial support. \\ Availability of data and materials \\ Not applicable \\ Authors' contributions \\ $\mathrm{OBH}$ and $\mathrm{MD}$ are responsible for the manuscript concept, portions of writing and editing. FD and MD are responsible for the collection of data and portions of writing. MMA and MD are responsible for the review of pathological findings. MK and MD are responsible for the manuscript concept, writing, and significant editing. All authors read and approved the} final manuscript.

Ethics approval and consent to participate

Not applicable

\section{Consent for publication}

All patients included in this study had signed an informed written consent for this information to be published (or if the patient is deceased or under 18 years old, the consent was signed by his/her next of kin or parent).

Competing interests

The authors declare that they have no competing interests.

\section{Publisher's Note}

Springer Nature remains neutral with regard to jurisdictional claims in published maps and institutional affiliations.

\section{Author details}

${ }^{1}$ Department of Neurosurgery, Firat University School of Medicine, Elazig, Turkey. ${ }^{2}$ Department of Pathology, Firat University School of Medicine, Elazig, Turkey.

Received: 9 October 2018 Accepted: 11 March 2019

Published online: 18 March 2019

\section{References}

1. Bell DF, Walker JL, O'Connor G, Tibshirani R. Spinal deformity after multiplelevel cervical laminectomy in children. Spine (Phila Pa 1976). 1994;19:40611.

2. Craig JB, Govender S. Neurofibromatosis of the cervical spine. A report of eight cases. J Bone Joint Surg Br. 1992;74:575-8. 
3. Crostelli M, Mariani M, Mazza O, Ascani E. Cervical fixation in the pediatric patient: our experience. Eur Spine J. 2009;18:20.

4. Friedman JM, Birch PH. Type 1 neurofibromatosis: a descriptive analysis of the disorder in 1,728 patients. Am J Med Genet. 1997;70:138-43.

5. Funasaki H, Winter RB, Lonstein JB, Denis F. Pathophysiology of spinal deformities in neurofibromatosis. An analysis of seventy-one patients who had curves associated with dystrophic changes. J Bone Joint Surg Am. 1994; 76:692-700.

6. Garg S, Hosalkar H, Dormans JP. Quadriplegia in a 10 year-old boy due to multiple cervical neurofibromas. Spine (Phila Pa 1976). 2003;28:E339-43.

7. Hioki A, Miyamoto K, Hirose Y, Kito Y, Fushimi K, Shimizu K. Cervical symmetric dumbbell ganglioneuromas causing severe paresis. Asian Spine J. 2014;8:74-8.

8. Kawabata S, Watanabe K, Hosogane N, Ishii K, Nakamura M, Toyama Y, et al. Surgical correction of severe cervical kyphosis in patients with neurofibromatosis type 1. J Neurosurg Spine. 2013;18:274-9.

9. McGirt MJ, Chaichana KL, Atiba A, Bydon A, Witham TF, Yao KC, et al. Incidence of spinal deformity after resection of intramedullary spinal cord tumors in children who underwent laminectomy compared with laminoplasty. J Neurosurg Pediatr. 2008;1:57-62.

10. McGirt MJ, Garcés-Ambrossi GL, Parker SL, Sciubba DM, Bydon A, Wolinksy JP, et al. Short-term progressive spinal deformity following laminoplasty versus laminectomy for resection of intradural spinal tumors: analysis of 238 patients. Neurosurgery. 2010;66:1005-12.

11. Miyakoshi N, Hongo M, Kasukawa Y, Misawa A, Shimada Y. Bilateral and symmetric C1-C2 dumbbell ganglioneuromas associated with neurofibromatosis type 1 causing severe spinal cord compression. Spine J. 2010;10:e11.

12. Tonogai I, Sakai T, Katoh S, Higashino K, Sairyo K, Hirohashi N, et al. Myelopathy in a 6-year-old girl caused by neurofibromatosis type 1: a case report. Spine J. 2008;8:836-40.

13. Ugarriza LF, Cabezudo JM, Ramirez JM, Lorenzana LM, Porras LF. Bilateral and symmetric $\mathrm{C} 1-\mathrm{C} 2$ dumbbell ganglioneuromas producing severe spinal cord compression. Surg Neurol. 2001;55:228-31.

14. Yong-Hing K, Kalamchi A, MacEwen GD. Cervical spine abnormalities in neurofibromatosis. J Bone Joint Surg Am. 1979;61:695-9.

\section{Submit your manuscript to a SpringerOpen ${ }^{\circ}$ journal and benefit from:}

- Convenient online submission

- Rigorous peer review

- Open access: articles freely available online

High visibility within the field

- Retaining the copyright to your article

Submit your next manuscript at $\boldsymbol{\nabla}$ springeropen.com 